\title{
Yield of Repeat 3D Angiography in Patients with Aneurysmal-Type Subarachnoid Hemorrhage
}

\author{
(D).S. Bechan, (DW.J. van Rooij, (D).P. Peluso, and (D) M. Sluzewski
}

\begin{abstract}
BACKGROUND AND PURPOSE: Aneurysmal-type subarachnoid hemorrhage is a serious disease with high morbidity and mortality. When no aneurysm is found, the patient remains at risk for rebleeding. Negative findings for SAH on angiography range from $2 \%$ to $24 \%$. Most previous studies were based on conventional $2 \mathrm{D}$ imaging. 3D rotational angiography depicts more aneurysms than $2 \mathrm{D}$ angiography. The purpose of this study was to evaluate the yield of repeat 3D rotational angiography in patients with aneurysmal-type SAH with negative initial 3D rotational angiography findings and to classify the initial occult aneurysms.
\end{abstract}

MATERIALS AND METHODS: Between March 2013 and January 2016, 292 patients with SAH and an aneurysmal bleeding pattern were admitted. Of these 292 patients, 30 (10.3\%; $95 \% \mathrm{Cl}, 7.3 \%-14.3 \%)$ had initial negative 3D rotational angiography findings within 24 hours. These patients underwent a second 3D rotational angiography after 7-10 days.

RESULTS: In 8 of 30 patients $(26.7 \%$; $95 \% \mathrm{Cl}, 14.0 \%-44.7 \%)$ with initial negative 3D rotational angiography findings, a ruptured aneurysm was found on repeat 3D rotational angiography. Three of 8 initial occult aneurysms were very small (1-2 mm), 2 were supraclinoid carotid artery dissecting aneurysms ( 2 and $8 \mathrm{~mm}$ ), 2 were small $(1$ and $3 \mathrm{~mm}$ ) basilar perforator aneurysms, and 1 was a 3-mm vertebral artery dissecting aneurysm.

CONCLUSIONS: In 10\% of patients with aneurysmal-type SAH, initial 3D rotational angiography findings were negative, and in 1 in 4 , repeat $3 \mathrm{D}$ rotational angiography demonstrated a ruptured aneurysm. Initial occult aneurysms were dissecting aneurysms of perforators or main arteries or were very small (1-2 mm) or both. Our results indicate that repeat 3D rotational angiography is mandatory in patients with initial 3D rotational angiography findings negative for aneurysmal-type SAH.

ABBREVIATIONS: aSAH = aneurysmal-type SAH; 3DRA $=3$ D rotational angiography

S ubarachnoid hemorrhage with an aneurysmal bleeding pattern (aSAH) is a serious disease with high morbidity and mortality. In $80 \%-90 \%$ of patients with aSAH, an aneurysm can be found as the cause of hemorrhage, ${ }^{1}$ and early repair is advocated to prevent recurrent hemorrhage.

The incidence of angiograms negative for aSAH has been reported to range from $2 \%$ to $24 \%$ in various studies. ${ }^{2,3}$ In some of these patients, the source of the hemorrhage is an occult aneurysm, but intracranial artery dissections, dural arteriovenous malformations, micro-AVMs, trauma, bleeding disorders, substance abuse, or other causes should also be considered. Even though no

Received March 27, 2016; accepted after revision July 8.

From the Department of Radiology, Sint Elisabeth Ziekenhuis, Tilburg, the Netherlands.

Please address correspondence to Willem Jan van Rooij, MD, Department of Radiology, Sint Elisabeth Ziekenhuis, Hilvarenbeekseweg 60, 5022GC Tilburg, the Netherlands; e-mail: wjjvanrooij@gmail.com

http://dx.doi.org/10.3174/ajnr.A4942 aneurysm is found, these patients are at risk for early rebleed with an inherent risk of morbidity and death. ${ }^{4}$ An aneurysm is not depicted on first angiography for several reasons: very small aneurysms, small aneurysms with an intraluminal thrombus, aneurysms on arterial dissections, suboptimal image quality due to technical reasons or in uncooperative patients, and a missed diagnosis of an aneurysm.

Repeat angiography is advocated to avoid missing a treatable cause of aSAH. Previous studies have demonstrated that repeat angiography after 7-10 days can depict an aneurysm in up to one-third of patients with initial negative angiography findings. $^{5-8} 3 \mathrm{D}$ rotational angiography (3DRA) has been proved to depict more aneurysms than 2D DSA. ${ }^{9,10}$ In our institution, diagnostic work-up of patients with aSAH now consists of 3DRA of all vessels within 24 hours. With negative findings, 3DRA is repeated. In this study, we evaluated the yield of repeat 3DRA in patients with aSAH with negative initial 3DRA findings. In addition, we classified the initial occult aneurysms. 
Types of aneurysms found on repeat 3DRA in patients with aSAH

\begin{tabular}{cclc}
\hline & Age (yr)/Sex & \multicolumn{1}{c}{ Aneurysm Location } & Size \\
\hline 1 & $61, \mathrm{M}$ & Dissecting aneurysm of perforator of the basilar tip & $3 \mathrm{~mm}$ \\
2 & $60, \mathrm{M}$ & Superior cerebellar artery & $1 \mathrm{~mm}$ \\
3 & $65, \mathrm{M}$ & Dissecting aneurysm of the supraclinoidal internal carotid artery & $2 \mathrm{~mm}$ \\
4 & $62, \mathrm{~F}$ & Dissecting aneurysm of the supraclinoidal internal carotid artery & $8 \mathrm{~mm}$ \\
5 & $46, \mathrm{~F}$ & Dissecting aneurysm of the V4 segment & $3 \mathrm{~mm}$ \\
6 & $62, \mathrm{~F}$ & Al-A2 junction & $1 \mathrm{~mm}$ \\
7 & $70, \mathrm{M}$ & A1 & $1 \mathrm{~mm}$ \\
8 & $65, \mathrm{M}$ & Dissecting aneurysm of perforator of the basilar tip & $1 \mathrm{~mm}$ \\
\hline
\end{tabular}

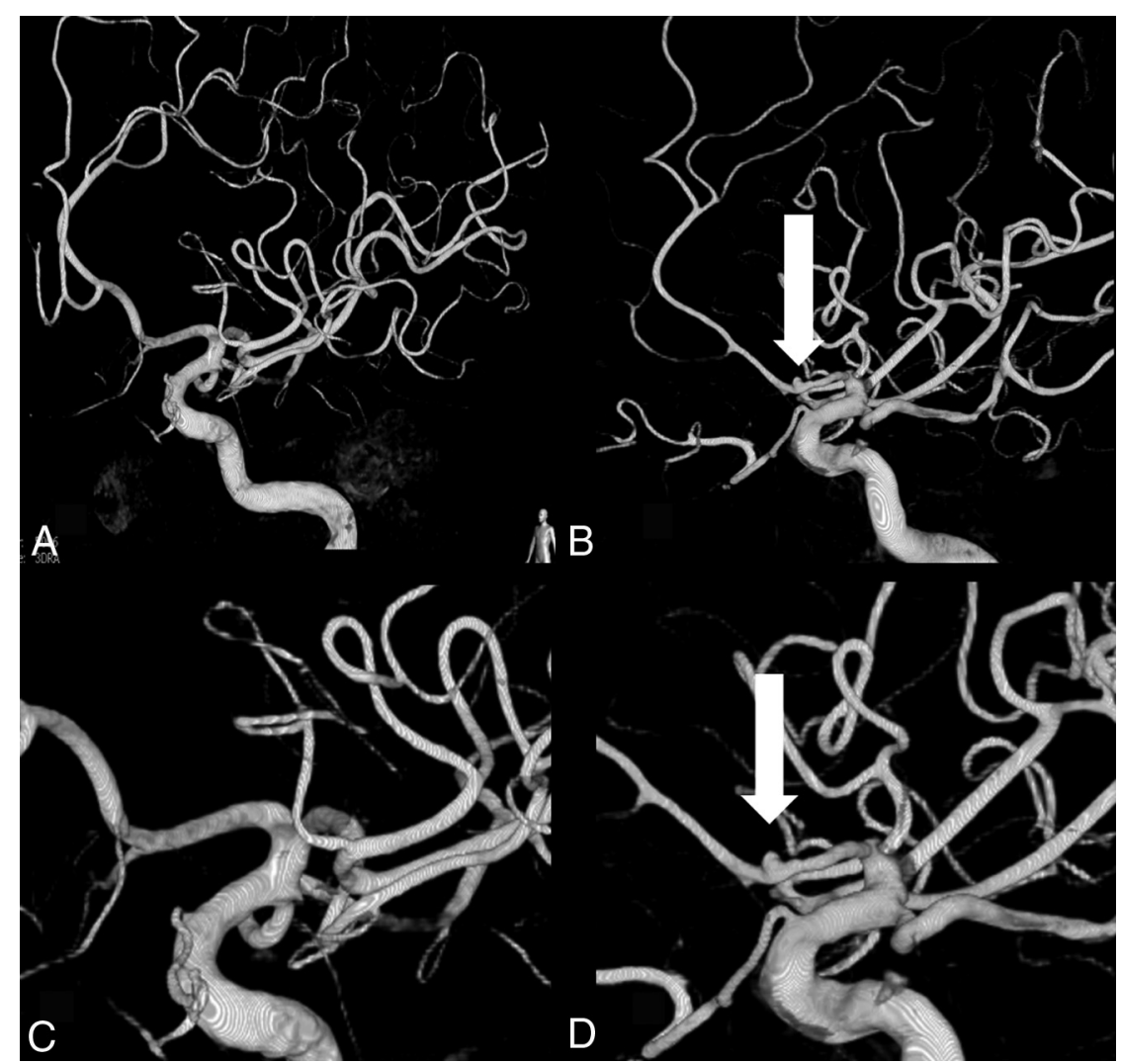

FIG 1. A 65-year-old man with initial negative findings on 3DRA. A, 3DRA within 24 hours after SAH shows no aneurysm. $B$, Repeat 3DRA after 10 days shows a very small $(2 \mathrm{~mm}) \mathrm{Al}$ aneurysm. $C$ and $D$, Magnification images of the Al segment and an Al aneurysm.

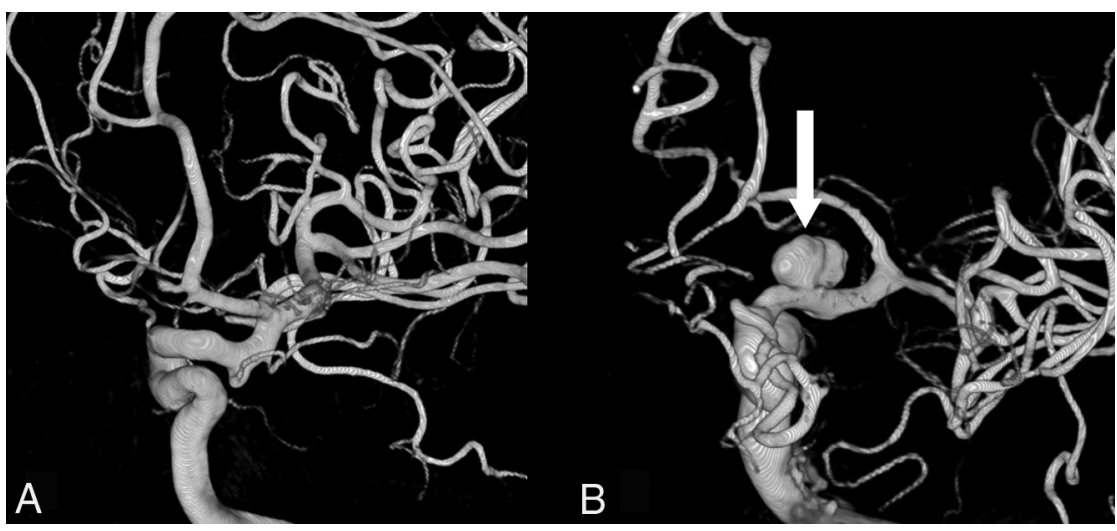

FIG 2. A 62-year-old woman with initial negative findings on 3DRA. A, 3DRA within 24 hours after SAH shows no aneurysm. $B$, Repeat 3DRA after 10 days shows an 8-mm supraclinoid internal artery dissecting aneurysm.

\section{MATERIALS AND METHODS}

\section{Patients}

This observational study with prospectively collected data was compliant with the institutional privacy policy. The institutional review board gave exempt status for approval and informed consent. Between March 2013 and January 2016, 292 patients with aSAH were admitted. The diagnosis of aSAH was established with CT. All 292 patients had 3DRA of all vessels within 24 hours. When the first 3DRA had negative findings, patients underwent a repeat 3DRA after 7-10 days.

\section{Analysis of 3D Rotational Angiography}

The protocol for 3D angiography has been described previously. ${ }^{11} 3 \mathrm{D}$ angiography examinations were reviewed by an experienced interventional neuroradiologist (W.J.v.R., with 27 years of experience) to determine the presence of a causative vascular abnormality. After review of the catheter angiograms, the presence of a causative vascular abnormality for the SAH was established by consensus of a panel comprising experienced interventional neuroradiologists and vascular neurosurgeons. Treatment decisions were also reached by consensus of this panel.

\section{Statistical Analysis}

Descriptive statistics were used for the presence, size, and locations of aneurysms. Quantitative variables were expressed with descriptive statistics, and categoric variables were expressed as frequencies or percentages with 95\% CIs. Statistical analysis was performed with MedCalc statistical software, Version 14.12.0 for Windows (MedCalc Software, Mariakerke, Belgium).

\section{RESULTS}

Of 292 patients admitted with aSAH on a native CT scan, in 30 patients $(10.3 \%$, 95\% CI, 7.3\%-14.3\%), no aneurysm was detected on 3DRA within 24 hours. In 8 of these 30 patients (27\%), an aneurysm was found on repeat 3DRA after 10 days. Qualification of these aneurysms is shown in the Table. Three of 8 initial occult aneurysms were very small (1-2 $\mathrm{mm}), 2$ were supraclinoid carotid artery dissecting aneurysms (2 and $8 \mathrm{~mm}$ ) 


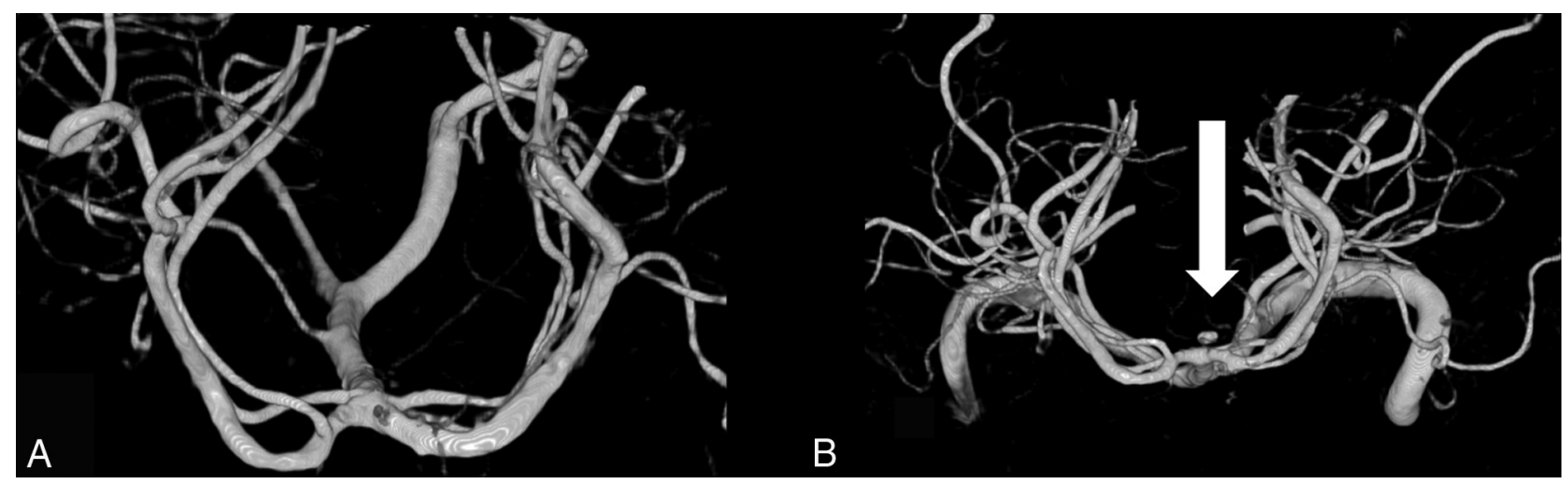

FIG 3. A 61-year-old man with initial negative findings on 3DRA. A, 3DRA (head-tail projection) within 24 hours after SAH shows no aneurysm. $B$, Repeat 3DRA (head-tail projection) after 10 days shows a very small basilar tip perforator dissecting aneurysm.

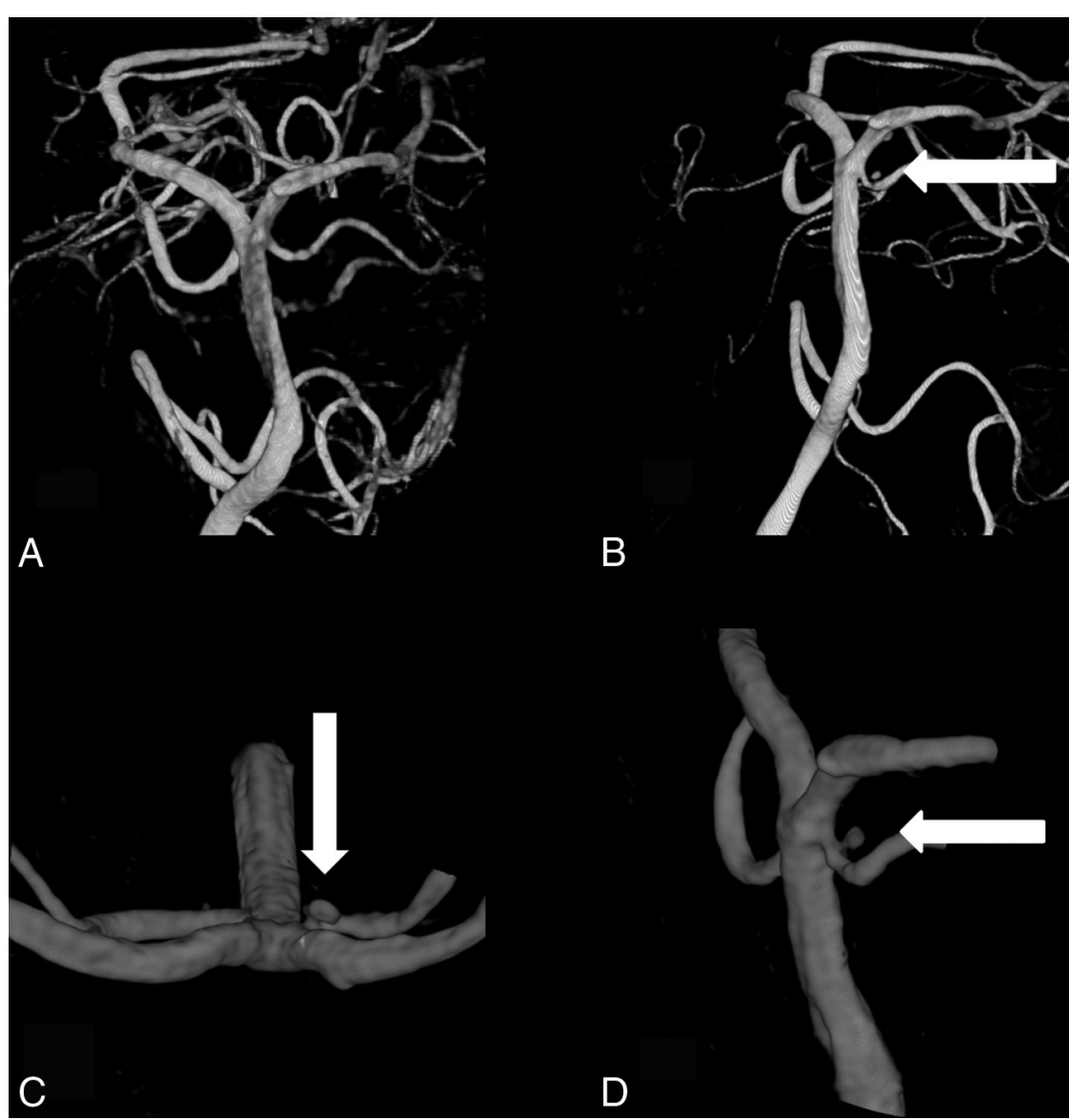

FIG 4. A 65-year-old man with initial negative findings on 3DRA. A, 3DRA within 24 hours after SAH shows no aneurysm. $B$, Repeat 3DRA after 10 days shows a very small basilar tip perforator dissecting aneurysm. C, Magnification image of a basilar tip perforator dissecting aneurysm (headtail projection). D, Magnification image of basilar tip perforator dissecting aneurysm (lateral projection).

(Figs 1 and 2), 2 were small (1 and $3 \mathrm{~mm}$ ) basilar perforator aneurysms (Figs 3 and 4), and 1 was a 3 -mm vertebral artery dissecting aneurysm (Fig 5). In retrospect, only one 1-mm A1 aneurysm was missed on initial 3DRA; the other aneurysms were not present at the time of first 3DRA.

\section{DISCUSSION}

The main cause of aSAH with an aneurysmal bleeding pattern is aneurysmal rupture, and angiography with false-negative findings to detect an aneurysm may result in poor patient outcome. Repeat imaging for the initial angiogram negative for aSAH is controversial because of the various diagnostic modalities and lack of a clear consensus on yield.

In a previous study, we demonstrated that selective 3DRA in addition to standard multiprojection $2 \mathrm{D}$ angiography yielded an aneurysm in almost $80 \%$ of patients (18 of 23 ) with $2 \mathrm{D}$ angiography negative for aSAH. The advantage of 3DRA over DSA is obvious: free rotation of high-resolution images in any projection without overprojecting bony structures. With 2D imaging, a small aneurysm may be obscured by overprojecting adjacent vessels in the limited number of available projections. ${ }^{10}$ Therefore, previous data of the yield of repeat 2D DSA cannot be compared with results of the yield of repeat 3DRA. To our knowledge, this is the first study using 3DRA for both initial and repeat angiography.

In the present study, we used 3DRA of all cerebral vessels as a standard diagnostic work-up. Despite this optimal vascular imaging, in $10 \%$ of patients (30 of 292) with aSAH, this was negative for aneurysms; and in 8 of these 30 patients $(27 \%)$, an aneurysm was found on repeat 3DRA. Not surprising, most initial occult aneurysms were very small, and most of them were, in retrospect, not visible on the initial 3DRA. Dissecting aneurysms of either a parent vessel or a perforator branch of the basilar artery composed the other types of aneurysms, which were probably not present on the initial 3DRA. Perforator artery aneurysms of the basilar trunk are rarely described in the literature. A recent literature review found 12 patients with basi- 


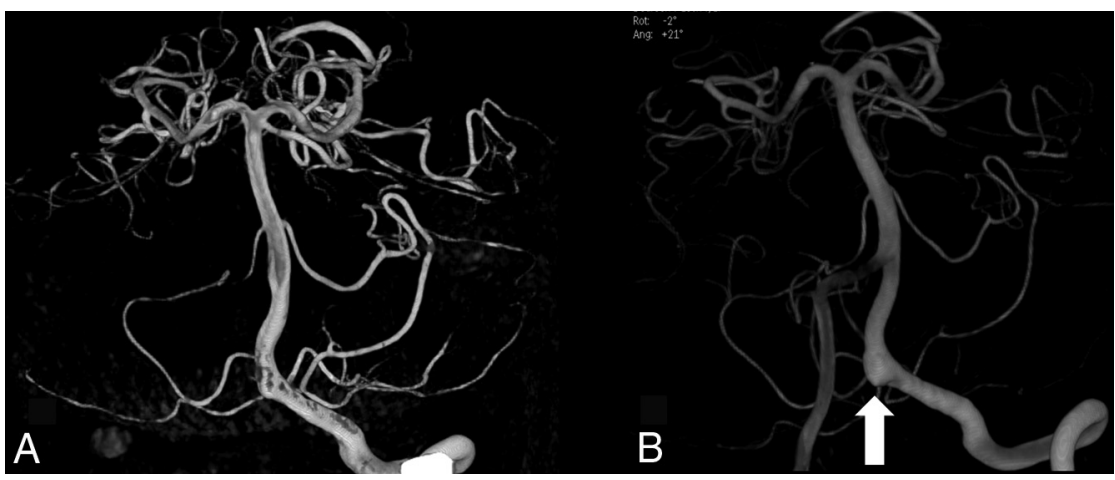

FIG 5. A 46-year-old woman with initial negative findings on 3DRA. A, 3DRA within 24 hours after SAH shows no aneurysm. $B$, Repeat 3DRA after 10 days shows a V4 dissecting aneurysm.

lar perforator artery aneurysms. All aneurysms arose from the middle or rostral basilar perforator arteries. ${ }^{12}$ The incidence of ruptured basilar perforator artery aneurysms in our study population was 2 of 270 ruptured aneurysms (0.7\%). Because many physicians are unfamiliar with these types of aneurysms, treatment poses a technical challenge.

A number of possible factors leading to no visualization of a structural lesion, when it is actually present, have been mentioned in the literature. These include thrombus inside the aneurysmal sac; compression by surrounding hematoma; focal arterial spasm, which may lead to a temporary obliteration of the aneurysm neck; and technical and interpretation factors. ${ }^{3,13,14}$

The timing of repeat angiography remains controversial. Randomized controlled trails do not exist to support any imaging protocol in the initial angiography negative for subarachnoid hemorrhage. However, data from large retrospective series provide useful information from the experience of a high-volume neurovascular center. ${ }^{15}$ In our hospital, repeat angiography is performed 7-10 days after the initial ictus, when the patient is still in the hospital. A study by Fontanella et $\mathrm{al}^{2}$ reported that the incidence of rebleed was 5\% and all the episodes occurred within 17 days after the initial ictus. Some authors advocate that repeat angiography be delayed 4-6 weeks after the initial ictus. ${ }^{15,16}$ The arguments in favor of delayed repeat angiography are that by this time, radiologic vasospasm has recovered and hematoma around the vessel and any thrombus inside the proximal vessel or aneurysm sac have also resolved, thus making visualization of the aneurysm easier. There are also authors who advocate even a third angiography; a large retrospective study reported a yield of $8 \%$ from a third angiography. ${ }^{15}$ In these previous studies, not all patients had a $3 \mathrm{D}$ reconstruction of the cerebral vessels. A large prospective study assessed the yield of repeat conventional angiography in patients with $\mathrm{SAH}$ and initial catheter angiograms with negative findings. The results of this study showed an overall yield of $4.2 \%$ for conventional angiography performed 7 days after presentation and $0 \%$ for conventional angiography performed 3 months after presentation. ${ }^{17}$ In our hospital, we decided not to do a third 3DRA.

\section{CONCLUSIONS}

Angiograms negative for aSAH are a very important clinical entity; the primary aim in management of these patients is to ensure that there is no structural lesion underlying the bleed, primarily aneurysms. The optimal imaging technique for depicting intracranial aneurysms is 3DRA. With this imaging quality, $10 \%$ of patients had an initial 3DRA with negative findings, and repeat 3DRA after 10 days revealed an aneurysm in 1 of 4 . These aneurysms were either very small or were dissecting aneurysms of either a large vessel or a perforator branch. Our results indicate that repeat 3DRA is mandatory in patients with initial negative findings.

\section{REFERENCES}

1. Byyny RL, Mower WR, Shum N, et al. Sensitivity of noncontrast cranial computed tomography for the emergency department diagnosis of subarachnoid hemorrhage. Ann Emerg Med 2008;51:697703 CrossRef Medline

2. Fontanella M, Rainero I, Panciani PP, et al. Subarachnoid hemorrhage and negative angiography: clinical course and long-term follow-up. Neurosurg Rev 2011;34:477-84 CrossRef Medline

3. Jung JY, Kim YB, Lee JW, et al. Spontaneous subarachnoid haemorrhage with negative initial angiography: a review of 143 cases. J Clin Neurosci 2006;13:1011-17 CrossRef Medline

4. Rinkel GJ, Wijdicks EF, Hasan D, et al. Outcome in patients with subarachnoid haemorrhage and negative angiography according to pattern of haemorrhage on computed tomography. Lancet 1991; 338:964-68 CrossRef Medline

5. Yu DW, Jung YJ, Choi BY, et al. Subarachnoid hemorrhage with negative baseline digital subtraction angiography: is repeat digital subtraction angiography necessary? J Cerebrovasc Endovasc Neurosurg 2012;14:210-15 CrossRef Medline

6. Khan N, Schuknecht B, Yonekawa Y. Presentation and management of patients with initial negative 4-vessel cerebral angiography in subarachnoid hemorrhage. Acta Neurochir Suppl 2002;82:71-81 Medline

7. Topcuoglu MA, Ogilvy CS, Carter BS, et al. Subarachnoid hemorrhage without evident cause on initial angiography studies: diagnostic yield of subsequent angiography and other neuroimaging tests. J Neurosurg 2003;98:1235-40 CrossRef Medline

8. Ishihara $\mathrm{H}$, Kato $\mathrm{S}$, Akimura $\mathrm{T}$, et al. Angiogram-negative subarachnoid hemorrhage in the era of three dimensional rotational angiography. J Clin Neurosci 2007;14:252-55 CrossRef Medline

9. van Rooij WJ, Sprengers ME, de Gast AN, et al. 3D rotational angiography: the new gold standard in the detection of additional intracranial aneurysms. AJNR Am J Neuroradiol 2008;29:976-79 CrossRef Medline

10. van Rooij WJ, Peluso JP, Sluzewski M, et al. Additional value of 3D rotational angiography in angiographically negative aneurysmal subarachnoid hemorrhage: how negative is negative? AJNR Am J Neuroradiol 2008;29:962-66 CrossRef Medline

11. Bechan RS, van Rooij SB, Sprengers ME at al. CT angiography versus 3D rotational angiography in patients with subarachnoid hemorrhage. Neuroradiology 2015;57:1239-46 CrossRef Medline

12. Gross BA, Puri AS, Du R. Basilar trunk perforator artery aneurysms: case report and literature review. Neurosurg Rev 2013;36:163-68; discussion 168 CrossRef Medline

13. Houben MP, van Rooij WJ, Sluzewski M, et al. Subarachnoid hem- 
orrhage without aneurysm on the angiogram: the value of repeat angiography [in Dutch]. Ned Tijdschr Geneeskd 2002;146:804-08 Medline

14. Kumar R, Kanti Das K, Sahu RK, et al. Angio negative spontaneous subarachnoid hemorrhage: is repeat angiogram required in all cases? Surg Neurol Int 2014;5:125 CrossRef Medline

15. Dalyai R, Chalouhi N, Theofanis T, et al. Subarachnoid hemorrhage with negative initial catheter angiography: a review of 254 cases evaluating patient clinical outcome and efficacy of short- and long- term repeat angiography. Neurosurgery 2013;72:646-52; discussion 651-52 CrossRef Medline

16. Gupta SK, Gupta R, Khosla VK, et al. Nonaneurysmal nonperimesencephalic subarachnoid hemorrhage: is it a benign entity? Surg Neurol 2009;71:566-67; discussion 571-72 CrossRef Medline

17. Delgado Almandoz JE, Jagdeesan BD, Refai D, et al. Diagnostic yield of repeat catheter angiography in patients with catheter and computer tomography angiography negative subarachnoid hemorrhage. Neurosurgery 2012;70:1135-42 CrossRef Medline 